\title{
Commentary
}

\section{Black In Neuro, Beyond One Week}

\author{
${ }^{\circledR}$ De-Shaine Murray, ${ }^{1}{ }^{\circledR}$ Lietsel Richardson, ${ }^{2}{ }^{\circledR}$ Rackeb Tesfaye, ${ }^{3}{ }^{\circledR}$ Danielle Nadin, ${ }^{4,5}{ }^{\circledR}$ Clíona Kelly, ${ }^{6}$ and \\ Paige Greenwood ${ }^{7,8}$ \\ ${ }^{1}$ Department of Bioengineering, Imperial College London, South Kensington, London, SW7 2AZ, United Kingdom, ${ }^{2}$ Department of Mechanical and \\ Aerospace, University of Central Florida, Orlando, Florida 32828, ${ }^{3} \mathrm{McGill}$ University Department of Neurology and Neurosurgery, Montreal \\ Neurological Institute, Montreal, Quebec H3A 2B4, Canada, ${ }^{4}$ Integrated Program in Neuroscience, Faculty of Medicine, McGill University, \\ Montreal, Quebec, H3A 0G4, Canada, ${ }^{5}$ Montreal General Hospital, McGill University Health Center Research Institute, Montreal, Quebec, H3G 1A4, \\ Canada, ${ }^{6}$ Aston Institute of Health and Neurodevelopment, School of Health and Life Sciences, Aston University, Birmingham, B4 7ET, United \\ Kingdom, ${ }^{7}$ University of Cincinnati, College of Medicine, Neuroscience Graduate Program, Cincinnati, Ohio 45267, and ${ }^{8}$ General Pediatrics, \\ Cincinnati Children's Hospital Medical Center, Cincinnati, Ohio 45229
}

\section{Introduction}

We at Black In Neuro have led a grassroots effort to empower Black scholars in neuroscience-related fields in 2020. Author and activist Audre Lorde said, "Revolution is not a one-time event." We call on our non-Black colleagues in neuroscience-related fields to mobilize and actively fight anti-Black racism. It is imperative that our community commit to ongoing reflection and action toward dismantling and rebuilding structures that have denied Black success, as well as building spaces that elevate and support Black scholars and trainees.

During a time filled with uncertainty because of coronavirus disease 2019 (COVID-19), another global crisis has taken center stage: anti-Black racism. The brutal injustice observed in 2020 is not new or unique to a specific geography. The disproportionate effect of COVID-19 on Black people combined with state-sanctioned violence has led to an unveiling of inequity that we experience across all facets of society. Black people face higher rates of police brutality, health complications, imprisonment, and poverty, and are subjected to lower rates of employment, homeownership, and college attainment across various countries (DeGue et al., 2016; Centers for Disease Control, 2017). As Black trainees, our lab coats, degrees, and accolades

\footnotetext{
Received Nov. 27, 2020; revised Jan. 2, 2021; accepted Jan. 6, 2021 The authors declare no competing financial interests.

Correspondence should be addressed to Paige Greenwood at blackinneuro@gmail.com.

https://doi.org/10.1523/JNEUROSCI.2957-20.2021

Copyright $(2021$ the authors
}

are not bulletproof and do absolutely nothing to protect us from systemic racism.

Black trainees and scientists carry these burdens while navigating uncertain prospects of academic careers, often in the face of isolation and alienation from our peers. Our Blackness was acutely felt this past summer as we received emails from our department heads, public statements on social media, and affirmations from our colleagues telling us that our lives mattered in the aftermath of George Floyd's murder. As we navigated conversations often centered around non-Black colleagues grappling with privilege, we were also asked to lead equity, diversity, and inclusion (EDI) efforts that have often been neglected. The latter isn't new. Underrepresented faculty disproportionately lead EDI efforts on campus, which hinders their advancement in the tenure process (Jimenez et al., 2019). This burden to create inclusive spaces is passed on generationally. Black faculty whose efforts have been dismissed by academic institutions are forced to pass on the torch of advocacy to Black trainees. \#BlackInNeuroWeek was born from the culmination of grief, isolation, and frustration with performative EDI efforts that we as science trainees have felt for many years.

Although we respect the overdue acknowledgment of anti-Black racism from our institutions and peers, we request meaningful action. We ask for white and non-Black people of color to confront their complicity in anti-Black racism. Black scientists do not have the luxury of existing in a world where science is apolitical. This May, in New
York Central Park, we witnessed a woman weaponize her whiteness against a Black bird watcher. She falsely reported to police that he had assaulted her, reminiscent of the lies that led to the senseless murder of Emmett Till in 1955. Galvanized by this event, and following the path paved by initiatives, such as \#BLACKandSTEM (D. N. Lee, 2014), Black scientists leveraged the reach of social media to create \#BlackBirdersWeek, which was followed by \#BlackBontanistsWeek and \#BlackInAstroWeek. Following their lead, 16 Black scientists and 6 allies from the United Kingdom, United States, and Canada created \#BlackInNeuro.

\section{Black In Neuro Week}

From Monday, July 27, 2020 to Sunday, August 2, 2020, \#BlackInNeuroWeek brought visibility to Black scientists in neuroscience-related fields and celebrated their often-overlooked contributions. As a team, we discussed the importance of going beyond the work of our respective institutions. Thus, the week encompassed five key themes: representation, education, access, community, and outreach. A website and accessible promotional media were released the week before, highlighting the organizers' vision and a call for the wider community to get involved.

\section{Representation}

On day one, we amplified and connected the wealth of Black talent that exists within the neuroscience community. We coined \#BlackNeuroRollCall to actively engage Black scholars on Twitter and 
Instagram. Black scientists across the world in neuroscience-related fields were placed front and center. Black individuals in the United States account for only 6\% of university faculty members, and these statistics are mirrored throughout the academic pipeline. In neuroscience-related fields specifically, SfN's 2017 NDP report placed the average percentage of Black $\mathrm{PhD}$ recipients at $4 \%$, whereas $3 \%$ of postdoctoral trainees and $1 \%$ of faculty in neuroscience were reported to be Black (Society for Neuroscience, 2017). This underrepresentation is seen in other countries too. Across the Atlantic, Black professors account for $<1 \%$ in the United Kingdom despite accounting for $>3 \%$ of the general United Kingdom population (Adams, 2020). In ad-? A3B2 re 3, j>dition, of 19,868 United Kingdom Research and Innovationfunded $\mathrm{PhD}$ studentships, only 245 were awarded to Black students (1.2\%) in the past 3 academic years (Williams et al., 2019). Despite operating in isolation, racially minoritized people are more likely to produce scientific innovations, which are often dismissed and do not translate to career advancement (Hofstra et al., 2020). Therefore, our Roll Call also served to celebrate the innovations and achievements in the Black In Neuro community.

\section{Education}

On Tuesday, July 28, 2020, we decided to inform and educate by highlighting the role of neuroscience in perpetuating and upholding racism, using the hashtag \#NeuroRacism. Topics of this nature are not typically featured in academic curricula, and many trainees advance without being taught how the outcomes of our scientific practices affect real people and have lasting moral and ethical implications. Through informative Twitter threads, an expert panel of Black professionals, and a journal club, which dissected articles in our field through a critical lens, we looked at historical, current, and potential biases that reside in and emerge from neuroscience. Highlighting topics ranging from phrenology to Artificial Intelligence, we discussed the outputs of our field in a wider societal context, emphasizing why science should not be conducted in a vacuum.

\section{Access}

Demystifying the journey scientists in our community have taken toward graduate studies and careers in neurosciencerelated fields is vital for increasing the recruitment and retention of Black people in neuroscience. Wednesday, July 29, 2020 brought \#BlackJourneyToNeuro, which provided a platform for clearly and transparently sharing the unique experiences of undergraduates, graduates, and career professionals in academia, industry, and science policy.

Within the theme of access, we wanted \#BlackInNeuroWeek to be accessible to as many members of the Black community as possible. Disability, and the Black disabled experience in particular, is seldom prioritized in academic settings (A. Lee, 2020). Prompted by our community, we were called on to confront our own ableism and erasure of the intersection between Blackness and disability. A member of our community coined the hashtag \#BlackAndDisabledInNeuro, and shared their personal experience in an article on our website, which was released on the 30th anniversary of the Americans with Disabilities Act (Nolan, 2020). In addition, we used professional live-captioning for our live events, added captions to our video content, and included alternative text on images shared on social media. Subsequently, we will endeavor to include the voices of Black and disabled neuroscientists in the future of our organization.

\section{Community and outreach}

We dedicated a day to artistic expression, highlighting the talent that Black scientists hold beyond the traditional boundaries set between science and art. Thursday, July 30, 2020 allowed Black In Neuro participants to show their creative side with \#BlackNeuroArt. From data visualizations to microscopy to art in the mediums of music, dance, hairstyles, and more, many shared their passions and were inspired by the creative outlets of their peers.

Toward the end of the week, we began to look to the future by highlighting the vital roles outreach and mentorship play in the recruitment, retention, and success of Black scientists. On Friday, July 31, 2020, \#BlackNeuroMentors highlighted Black-led initiatives with a roundtable discussion from community members who have gone above and beyond to nurture students. Additionally, we partnered with Skype a Scientist to host \#SkypeABlackNeuroscientist, where elementary, middle school, and high school kids were able to pose questions to experts in our field. We felt it was important for younger generations of all backgrounds to see and interact with Black neuroscientists. Over the course of the day, participants were also encouraged to seek mentors and mentees. Mentorship is crucial for success and retention in academia, but even more so for students of color, who are less likely to pursue academic careers than their white counterparts (58.4\% vs 62.4\%) after graduation (Brunsma et al., 2017). Our goal was to begin closing that gap, by providing access to mentors.

Our weekend activities began on Saturday, August 1, 2020, by amplifying Black women within our field. Marginalization on the basis of race is difficult enough, but Black women also face discrimination on the grounds of gender. Beautifully coinciding with the birthday of Henrietta Lacks, \#BlackWomenInNeuro focused on the voices of Black women, fostering cohesiveness through a virtual social. Not only women are marginalized on the basis of their gender. We therefore invited nonbinary and trans members of our community to participate, recognizing that future events dedicated to gender nonconforming Black In Neuro members are required. This day reinforced the importance of not viewing Black people as a monolith. We have intersecting identities, which result in unique experiences that need to be respected by our institutions and society at large.

On Sunday, August 2, 2020, we centered joy to conclude our proceedings. The community was asked to share images of things that made them happy under the hashtag \#BlackJoyInNeuro. We supplemented this with a community dance class via Instagram live and the sharing of resources pertaining to Black mental health, as conversations surrounding mental health in the Black community have been steeped with stigma, along with barriers to accessing culturally appropriate services. As a community that has been subjected to such negativity and grief, both historically and in the present day, having an outward celebration of unadulterated Black happiness brought a perfect ending to our campaign.

\section{Outcomes and impact}

Before the start of \#BlackInNeuroWeek, we collected $>300$ profiles of international Black scientists in neuroscience-related fields on www.BlackInNeuro.com. By providing a visible database of Black trainees, scientists, and professionals, we have created a resource to help the Black In Neuro community connect. More importantly, it represents the large and talented pool of Black In Neuro members that are 
often overlooked for career opportunities. We also gauged our interaction with the community across social media. Even before \#BlackInNeuroWeek began, >1.5 million people from around the world interacted with our content on both Twitter and Instagram. On the first day of \#BlackInNeuroWeek, 1.5 million people engaged with our content, with $>3$ million views across the two platforms. Over the week, we had Twitter engagement rates that were 30 times the industry standard, with links to resources clicked hundreds of times per day. Content from the @BlackInNeuro Twitter account alone received 3.4 million impressions during the week. In addition, there were countless stories of the formation of new friendships and collaborations, and of many scientists creating social media accounts solely to participate.

Directly following \#BlackInNeuroWeek, we observed the positive impact of our database through feedback received from community members. Our website holds extensive resources for undergraduate, graduate, and postdoctoral scholars, including mentorship, outreach, and funding opportunities. These were collated during \#BlackInNeuroWeek for our community and continue to be highly engaged with and added to by our members. We have also reached $>500$ profile submissions for our website and plan to document the continuing impact of our initiative more formally. Overall, our efforts showed the power of inclusive grassroot initiatives and the communities they can develop, and resulted in features in STAT News, USA Today, and Forbes.

\section{Vision and call to action}

After a week of mass online engagement by the Black community and our nonBlack allies, many of us returned to our institutions and laboratories with hope. However, since \#BlackInNeuroWeek, we have witnessed more accounts of violence against Black people and watched our institutions try to return to "normal" without addressing their previous public statements of solidarity. Public support for the Black Lives Matter Movement is in decline (Pan and Moore, 2020). Fatigue among non-Black people engaging with anti-Black racism is increasing, but we do not get to turn off our Blackness to exist in our laboratories and offices. This fight is long-term, and it will be difficult. As the world rebuilds from this pandemic, this is a pivotal moment for the scientific community to mobilize and reconstruct spaces that have long been beneficiaries of antiBlack racism. To that end, we ask all members of our neuroscience community to join Black In Neuro, as we move beyond a week to support Black scientists by providing representation, education, access, community, and outreach on an ongoing basis. We are committed to improving the equity, diversity, inclusivity and accessibility of neurosciencerelated fields.

Capitalizing on the momentum of Black In Neuro Week, we have continued to address the needs of our community by hosting social gatherings, panels, and workshops on a range of topics relevant to the success of Black scientists in neuroscience-related fields. We did this most recently with our Black In Neuro Mini Conference (October 31, 2020 through November 4, 2020; \#BiNConference2020), which was preceded by a selection of curated events in collaboration with Neuromatch. We had $>1000$ registrants from 48 different countries sign up for our free virtual events. All were welcome to attend this celebration of neuroscience research undertaken by Black researchers through keynote speeches and data blitz talks, which allowed trainees to expand their CVs, despite the barriers and restrictions that have arisen because of COVID-19.

Because of the growing profiles database on our website, Black In Neuro also has the potential for establishing a mentorship program. We want to empower, inspire, and uplift other Black scientists by providing a safe virtual, and hopefully physical, space for the Black In Neuro community to grow. Our goal is for Black graduates, postgraduates, and non-Black allies to interact, collaborate, and foster meaningful relationships. However, for Black In Neuro to succeed, we need our communities' help. Just as glial cells play an active, and not passive, role in the functioning, protection, and nourishment of neurons, we are calling on individuals and institutions to support our development by the following:

1. Investing time, money, and resources. Collaborate with us to create free training opportunities, such as internships or summer research programs, for Black trainees at all levels.

2. Being mentors. Provide advice, support, and resources for Black students at various career stages. We encourage you to provide opportunities to students who may not fit traditional metrics of success (e.g., GPA, GRE), because these metrics have not only been shown to be a poor indicator of graduate school success, but also reinforce systemic barriers that Black trainees disproportionately face.

3. Compensating. Ensure Black trainees and colleagues are being compensated and rewarded for their EDI efforts.

4. Revising your curricula. Whether for a course or journal club, reflect on the diversity of your author list and actively discuss racial biases in your work and course content.

5. Being accountable. Call out instances of injustice and help create environments that allow us to excel with our science.

Further updates on our efforts and the future of this organization can be found at www.BlackInNeuro.com. From here you can register for events and receive the latest news on our development. We stress that we would like all members of the neuroscience community to collaborate and engage with us and respect that we will be centering the development and experiences of Black people within our field.

Overall, we are elated with the impact of \#BlackInNeuroWeek and its success in such a short period of time. In $<1$ month, 22 people at various stages of their training set a blueprint for the long-term enfranchisement of the Black community in academia. But we don't just want more Black talent to enter the academy. Rather, we want them to remain and excel once there. To actively combat the entrenched anti-Black racism in our institutions and create meaningful change, we must all extend our efforts beyond one week.

We dedicate this piece to the countless Black lives lost to injustice in 2020. We speak your names and fight for you every day. We wish to thank the entire Black In Neuro team for their passion and continued commitment and hard work in service to this cause. The founding Black In Neuro Team: Destinee Aponte, Thiago Arzua, Ashley Cunningham, Elena Dominguez, Angeline Dukes, Brielle Ferguson, Paige Greenwood, Monica Javidnia, Clíona Kelly, Christine Liu, De-Shaine Murray, Danielle Nadin, D'Angela Pritchett-Rowe, Stephanie Renee, Lietsel Richardson, Ti'Air Riggins, Ubadah Sabbagh, Cheryl Santa Maria, Kaela Singleton, Rackeb Tesfaye, Gwenaëlle Thomas, and Samantha Yammine. We also thank the broader neuroscience community, individuals and institutions, for being a part of this movement and supporting its launching. 


\section{References}

Adams R (2020) Fewer than 1\% of UK university professors are black, figures show. The Guardian.

Brunsma DL, Embrick DG, Shin JH (2017) Graduate students of color: race, racism, and mentoring in the white waters of academia. Sociol Race Ethnicity 3:1-13.

Centers for Disease Control (2017) African American health. Retrieved November 30, 2020. https://www.cdc.gov/vitalsigns/aahealth/ index.html.

DeGue S, Fowler KA, Calkins C (2016) Deaths due to use of lethal force by law enforcement: findings from the national violent death reporting system, 17 US states, 2009-2012. Am J Prev Med 51:S173-S187.

Hofstra B, Kulkarni VV, Galvez SM, He B, Jurafsky D, McFarland DA (2020) The diversity-innovation paradox in science. Proc Natl Acad Sci USA 117:9284-9291.

Jimenez MF, Laverty TM, Bombaci SP, Wilkins K, Bennett DE, Pejchar L (2019) Underrepresented faculty play a disproportionate role in advancing diversity and inclusion. Nat Ecol Evol 3:10301033.

Lee A (2020) A forgotten underrepresented group: students with disabilities' entrance into STEM fields. Intl J Disabil Dev Educ $1-18$.

Lee DN (2014) You should know: Stephani Page and \#BLACKandSTEM. Retrieved December 31, 2020. https://blogs.scientificamerican.com/ urban-scientist/you-should-know-stephani-pageand-blackandstem/.

Nolan S (2020) I am black and disabled, hear me. Retrieved December 31, 2020. https://www. black inneuro.com/perspectives/i-am-black-anddisabled.

Pan D, Moore D (2020) A new survey says white support for Black Lives Matter has slipped: some historians say they're not surprised. The Boston Globe. Retrieved November 30, 2020. https:// www.bostonglobe.com/2020/09/24/metro/ new-survey-says-white-support-black-livesmatter-has-slipped-some-historians-say-theyrenot-surprised/.

Society for Neuroscience (2017) Report of Neuroscience Departments \& Programs Survey. Retrieved November 30, 2020. https:// www.sfn.org/-/media/SAN/Documents/SurveyReports/NDP-Final-Report.ashx?la=en.

Williams P, Bath S, Arday J, Lewis C (2019) The broken pipeline: barriers to Black $\mathrm{PhD}$ students accessing Research Council funding: Leading Routes. www.leadingroutes. org. 